\title{
e-Phaïstos
}

e-Phaïstos

Revue d'histoire des techniques / Journal of the history

of technology

IV-1 | 2015

Les arts de guerre et de grâce (XIVe-XVIIle siècles)

\section{Analyse comparée de l'escrime, des barres et du jeu de paume}

A comparative analysis of fencing, jeu des barres, and jeu de paume

\section{Olivier Dupuis}

\section{(2) OpenEdition \\ Journals}

Édition électronique

URL : http://journals.openedition.org/ephaistos/626

DOI : 10.4000/ephaistos.626

ISSN : 2552-0741

Éditeur

IHMC - Institut d'histoire moderne et contemporaine (UMR 8066)

Édition imprimée

Date de publication : 1 avril 2015

Pagination : 27-42

ISSN : 2262-7340

Référence électronique

Olivier Dupuis, «Analyse comparée de l'escrime, des barres et du jeu de paume », e-Phaïstos [En ligne], IV-1 | 2015, mis en ligne le 22 novembre 2016, consulté le 20 avril 2019. URL : http:// journals.openedition.org/ephaistos/626 ; DOI : 10.4000/ephaistos.626 


\title{
Analyse comparée de l'escrime, des barres et du jeu de paume
}

\author{
Olivier Dupuis \\ Chercheur indépendant
}

Les conduites motrices peuvent se dérouler et se concevoir indépendamment des relations à autrui, mais dans de nombreuses situations, les interactions entre les individus agissent comme vecteur essentiel de leurs actions, que ce soit en collaboration ou en opposition. Parlebas désigne par le terme de « sociomotricité » la catégorie des activités pour lesquelles la communication motrice est essentielle et propose plusieurs outils permettant d'analyser les jeux sportifs sous cet angle ${ }^{1}$. De la sociomotricité à la sémiotricité ${ }^{2}$, il n'y a qu'un pas : c'est grâce aux signes produits par le comportement des partenaires ou des adversaires qu'un participant décide de la conduite à tenir. Ceci s'applique tout aussi bien pour des jeux sportifs attestés sous l'Ancien Régime. L'escrime, le jeu de paume et le jeu des barres serviront d'exemples pertinents pour cette période. En effet ces activités existent en Europe, et notamment en France, du XVe au XVIII e siècle et s'adressent à une large frange de la population comme le montre bien la répartition sociologique des joueurs par type de jeu exposée par Jean-Michel Mehl3. Si statistiquement la noblesse est peu représentée parmi les joueurs, son attachement aux activités physiques de loisir est bien attestée en tant "qu'elles permettent aux aristocrates de faire la preuve de qualités individuelles réelles, en harmonie avec les valeurs prééminentes du second ordre médiéval : vaillance, hardiesse, prouesse, largesse, force et droiture $\gg 4$.

La pratique de l'escrime en tant que jeu est attestée depuis le Moyen Âge ${ }^{5}$; la préparation au duel à armes réelles restait sans conteste une motivation permanente jusqu'au XvIII ${ }^{\mathrm{e}}$ siècle ${ }^{6}$, il n'en reste pas moins que l'art des armes servit aussi à l'éducation du corps et de loisir. Ces jeux restent assez peu règlementés, à l'exception de compétitions locales, ou jeux de prix, dont quelques règlements nous sont parvenus du XVe siècle au XVIII ${ }^{\mathrm{e}}$ siècle. Par exemple, la confrérie des escrimeurs lillois a rédigé vers 1600 le règlement d'une compétition annuelle permettant de désigner le « roi » de la confrérie 7 . Le précédent roi endosse alors le rôle de défenseur. Chaque participant dispose de trois opportunités ou «venues » de s'opposer au défenseur en place, la venue, temps de base du combat, se termine lorsqu'un des protagonistes touche l'autre au-dessus de la ceinture. De fait plutôt que d'interdire les touches simultanées, le règlement prévoit que dans un tel cas, le point revienne systématiquement au défenseur. Si c'est le "venant » qui réussit à toucher le défenseur sans être touché en retour, celui-ci endosse alors à son tour le rôle de défenseur. Le nouveau roi est le dernier défenseur en place. Cette règle de jeu instaure des relations asymétriques entre les participants n'est pas si éloignées de certaines règles de tournois médiévaux qui distinguaient les assaillants des défenseurs, comme par exemple celui de Chauvency, en $1285^{8}$.

Le jeu de paume apparaît clairement dans les

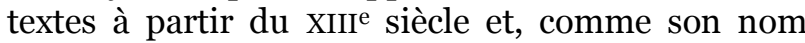
l'indique, s'est d'abord pratiqué à main nue ou gantée, avant que n'apparaissent les raquettes vers 1500. Comme tout jeu populaire, il est multiforme, se jouant en plein champ ou en ville contre les murs, sur les toits, seul ou opposant deux équipes de deux à six joueurs 9 . L'objectif est de chasser la balle de son camp et de l'envoyer dans le camp ad- 
verse, d'où la balle est ensuite " rechassée », et ainsi de suite jusqu'à ce que la balle ne puisse être renvoyée avant un second rebond. Dans ce cas, la " chasse » est marquée soit à l'emplacement du second rebond dans les jeux se tenant en salle, soit où la balle est arrêtée après ce second rebond pour les jeux en plein air. À ce stade, le point n'est pas encore attribué, l'équipe au service passe côté rachat, et l'équipe qui était au rachat passe côté service. Si cette dernière réussit aussi une chasse, le point est accordé à l'équipe qui aura fait la chasse la plus éloignée du filet, sinon il revient à l'équipe ayant réussi la première chasse. Parmi les nombreux jeux dérivés ${ }^{10}$, seul le jeu de courte paume, c'est-à-dire de jeu en salle fermée, et à la raquette, est suffisamment documenté depuis le $\mathrm{Xv}^{\mathrm{e}}$ siècle pour permettre d'en discuter les règles. Sa pratique en salle se répand dans toute l'Europe ${ }^{11}$, probablement porté par l'engouement de la bourgeoisie et la noblesse pour ce passe-temps, avec plusieurs combinaisons de joueurs (un contre un, un contre deux, etc., jusqu'à trois contre trois). La courte paume jouée en salle à un contre un sera la forme retenue pour la comparaison avec les autres jeux. Pour plus de clarté, la figure 1 présente le schéma d'une salle de jeu de paume à dedans, l'un des deux types de salle.

La définition du jeu des barres du dictionnaire de Richelet de 1690 le définit comme un "jeu où deux troupes de jeunes gens se rangeant en haie à la tête, \& à quelque distance les uns des autres sortent de leur rang, \& courant les uns après les autres tâchent de s'attraper \& de se faire prisonniers. ». Il s'agit d'un jeu d'attrape où le but est de faire prisonnier tous les joueurs du camp adverse sous réserve de respecter les contraintes suivantes :

- tout joueur entrant dans l'espace de jeu ne peut faire prisonnier que le dernier adversaire étant entré dans l'espace de jeu, l'expression consacrée est qu'il a «barre sur lui »;

- tout joueur de cet espace peut libérer l'ensemble des prisonniers de son camp.

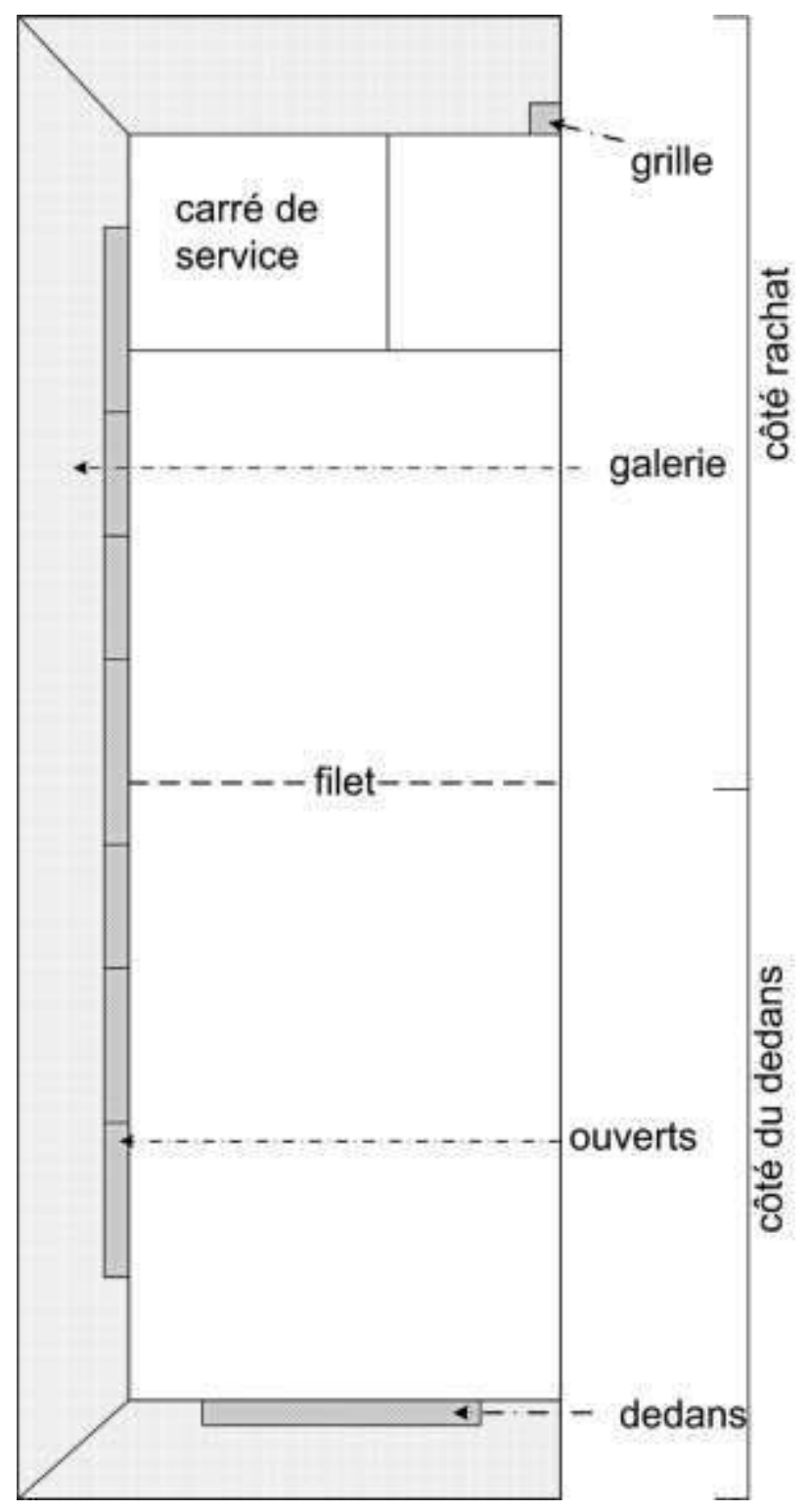

Figure 1 : Schéma d'une salle de jeu de paume à dedans.

L'origine du terme est incertain et pourrait être associé aux barrières qui auraient limité l'espace de 
jeu des joueurs ; en tout état de cause il apparaît vers la fin du XIII ${ }^{\mathrm{e}}$ siècle mais pourrait avoir été pratiqué bien avant d'après Mehl ${ }^{12}$. À un lecteur moderne, ce jeu semble tout fait pour la jeunesse, et de fait, cette activité entre couramment dans le programme d'éducation de la noblesse. Olivier de la Marche mentionne par exemple que le jeune comte de Charolais, le futur duc de Bourgogne Charles le Téméraire "jouoit aux barres à la façon de Picardie et escouoit les autres par terre et loin de luy ${ }^{13}$. Ce jeu attirait aussi le monde adulte au moins jusqu'au XVIII ${ }^{\mathrm{e}}$ siècle ${ }^{14}$, et non seulement celui des basses couches sociales mais aussi ces jeunes aristocrates français en exil à Gènes en $1797^{15}$, bien que rien ne permette de garantir que la forme jouée soit exactement la même. Cette activité était clairement entendue comme un mimétisme de la guerre, jusqu'à le voir conseiller comme exercice pour les soldats à la toute fin du XVIII ${ }^{\mathrm{e}}$ siècle :

« on sait qu'il est utile de rendre le soldat agile \& léger à la course ; que ce n'est que par des exercices violens qu'on y parvient; on sait que le jeu des barres est un des plus propres à remplir ces différens objets, \& cependant on ne le fait jamais jouer aux soldats : cet oubli, ou pour mieux dire, cette incurie, étonne tout observateur attentif.»16

Bascetta distingue les formes écrites traitant de l'escrime entre celles décrivant l'exécution, incluant les illustrations, et celles contenant le code verbal incluant les traités didactiques et les traités de perfectionnement ${ }^{17}$. À ce titre, le jeu des barres est le parent pauvre puisque hormis un petit fascicule de quatre pages imprimé en $1719^{18}$, entrant probablement dans les codes verbaux normatifs, aucune production écrite de code verbal n'est connue avant que ce jeu violent mimant la guerre et les tournois ne soit cantonné qu'aux jeux d'enfant au XIXe siècle. Pourtant les descriptions qu'en donnent les textes permettent d'entrapercevoir les rôles sociomoteurs et au moins schématiquement les stratégies pouvant être mises en jeu. Notre connaissance des modalités de jeu reste très lacunaire, ce qui nuit fortement à toute recréation poussée. Le premier écrit normatif concernant le jeu de paume date de 1555 et récapitule les règles de différents modes de jeu, décrit les caractéristiques attendues des terrains de jeu, des raquettes et des balles ${ }^{19}$. C'est donc déjà un jeu très civilisé qui malgré son succès auprès des gens aisés, ne fit l'objet que de très peu de textes didactiques ou technico-tactiques; à vrai dire, le seul rentrant clairement dans cette catégorie pour la période nous intéressant est un petit chapitre secondaire d'un livre d'escrime de $1573^{20}$ ! Â l'opposée, les jeux d'armes disposent eux d'une vaste panoplie de traités de perfectionnement, rarement didactiques. Il n'y a pour ainsi dire presque jamais de description du cadre de production des aptitudes décrites et encore moins des codes. Lors des essais de restitution des gestes, il n'est pas rare que des situations non documentées se présentent sans qu'il ne soit possible de savoir en quoi elles sont impertinentes et n'ont pas été retenues par l'auteur.

Cette forte diversité dans la mise par écrit du savoir-faire interpelle; si cette problématique va bien au-delà du sujet de cet article, elle montre au moins que la comparaison entre les activités motrices nécessite de disposer d'autres outils que les seuls instructions écrites.

Comme l'indique Parlebas, la modélisation mathématique offre des moyens permettant d'étudier la dynamique des actions corporelles, en particulier dans le cadre des communications motrices de manière rigoureuse et sans se cantonner à l'inventaire des comportements ludiques ${ }^{21}$. Cette méthode permet de surcroît de comparer des jeux en apparence fort dissemblables ${ }^{22}$, mais aussi de compléter leur étude, en particulier pour des jeux dont l'usage a complètement disparu de nos jours et qu'il est particulièrement difficile en conséquence de recréer.

Le statut sociomoteur d'un joueur à un moment donné de la partie correspond à l'ensemble des contraintes, des droits et des interdits prescrits à ce joueur par les règles du jeu sportif considéré. Il peut être décrit et distingué des autres statuts par les caractéristiques suivantes :

- relation à autrui ; 
- $\quad$ relation au temps ;

- relation à l'espace ;

- relation aux objets.

Pour la courte paume, ceci donne le tableau cidessous $^{23}$.

Pour le jeu des barres, seuls les rôles attestés avant le XVIII ${ }^{e}$ siècle ont été retenus. Je n'ai pas retrouvé en particulier de mention du rôle « en campagne » avant le XIX ${ }^{\mathrm{e}}$ siècle, en conséquence ce dernier a été écarté. Cependant, malgré la méconnaissance des modalités précises de défi et de capture, ceci n'entrave pas la description des rôles sociomoteurs.

\begin{tabular}{|c|c|c|c|c|}
\hline Rôle & Autrui & Espace & Temps & Objets \\
\hline Bailleur & $\begin{array}{l}\text { Lance la balle. } \\
\text { Interaction en fonction } \\
\text { du placement et de } \\
\text { l'attitude }\end{array}$ & $\begin{array}{l}\text { Le bailleur se place du côté du } \\
\text { dedans. La balle doit rouler sur } \\
\text { le toit et tomber dans le carré de } \\
\text { service }\end{array}$ & $\begin{array}{c}\text { Changement de } \\
\text { bailleur à chaque } \\
\text { chasse. }\end{array}$ & $\begin{array}{c}\text { Lance la balle et } \\
\text { la frappe }\end{array}$ \\
\hline Rechasseur & \multirow{3}{*}{$\begin{array}{l}\text { Renvoie la balle. } \\
\text { Interaction en fonction } \\
\text { du placement et de } \\
\text { l'attitude }\end{array}$} & $\begin{array}{l}\text { Se place à proximité du carré de } \\
\text { service. Renvoie la balle dans le } \\
\text { terrain adverse par-dessus le } \\
\text { filet. }\end{array}$ & $\begin{array}{l}\text { Changement de } \\
\text { côté à chaque } \\
\text { chasse. }\end{array}$ & $\begin{array}{l}\text { Tape la balle } \\
\text { avant deux } \\
\text { rebonds à sa } \\
\text { descente du toit. }\end{array}$ \\
\hline $\begin{array}{l}\text { Joueur } \\
\text { d'échange } \\
\text { côté du } \\
\text { dedans }\end{array}$ & & $\begin{array}{l}\text { Se place où il veut. Renvoie la } \\
\text { balle sur le carreau, sur les toits, } \\
\text { dans les galeries. Marquage des } \\
\text { chasses si deux rebonds. }\end{array}$ & \multirow{2}{*}{ Continuité du jeu } & \multirow{2}{*}{$\begin{array}{c}\text { Tape avant ou } \\
\text { après un rebond } \\
\text { au sol de la balle. } \\
\text { Si deux rebonds, } \\
\text { il y a chasse. }\end{array}$} \\
\hline $\begin{array}{l}\text { Joueur } \\
\text { d'échange } \\
\text { côté rachat }\end{array}$ & & $\begin{array}{c}\text { Comme précédemment }+ \\
\text { renvoie la balle dans la grille ou } \\
\text { la planche. }\end{array}$ & & \\
\hline
\end{tabular}




\begin{tabular}{|c|c|c|c|}
\hline Rôle & Autrui & Espace & Temps \\
\hline Prisonnier & Peut être délivré & $\begin{array}{c}\text { À côté du camp } \\
\text { adverse }\end{array}$ & $\begin{array}{c}\text { Délivré après qu'un partenaire ait } \\
\text { touché le dernier dans la chaîne des } \\
\text { prisonniers }\end{array}$ \\
\hline En attente & $\begin{array}{c}\text { Ne peut être capturé, ni capturer ni } \\
\text { libérer } \\
\text { Peut être défié }\end{array}$ & Se tient dans son camp & $\begin{array}{c}\text { Attend d'être défié ou qu'un nouvel } \\
\text { adversaire entre dans le champ }\end{array}$ \\
\hline $\begin{array}{c}\text { Joueur de } \\
\text { champ }\end{array}$ & $\begin{array}{c}\text { Annce "J'ai barre sur..." } \\
\text { Péfie un adversaire en lui frappant } \\
\text { dans la main } \\
\text { capturer le joueur adverse sur } \\
\text { lequel il a barre } \\
\text { Peut être poursuivi par le joueur } \\
\text { adverse qui a barre sur lui } \\
\text { Peut tenter de libérer les } \\
\text { prisonniers }\end{array}$ & $\begin{array}{c}\text { Dans le terrain de jeu. } \\
\text { Peut sortir du terrain } \\
\text { de jeu pour regagner } \\
\text { son camp }\end{array}$ & $\begin{array}{c}\text { Ce n'est qu'après être rentré sur le } \\
\text { champ qu'un joueur adverse peut } \\
\text { rentrer avoir barre sur soi }\end{array}$ \\
\hline
\end{tabular}




\begin{tabular}{|c|c|c|c|c|}
\hline Rôle & Autrui & Espace & Temps & Objet \\
\hline Défenseur & $\begin{array}{l}\text { Affronte le venant et tente de } \\
\text { garder son rôle } \\
\text { A le droit d'effectuer un coup } \\
\text { fourré }\end{array}$ & $\begin{array}{l}\text { Reste dans l'espace de } \\
\text { combat tant qu'il ne } \\
\text { perd pas de venue }\end{array}$ & & \multirow{2}{*}{$\begin{array}{l}\text { La touche } \\
\text { s'effectue au- } \\
\text { dessus de la } \\
\text { ceinture de } \\
\text { l'adversaire }\end{array}$} \\
\hline Venant & $\begin{array}{l}\text { Affronte le défenseur et } \\
\text { cherche à prendre sa place }\end{array}$ & $\begin{array}{l}\text { Dans l'espace de combat } \\
\text { durant la venue }\end{array}$ & $\begin{array}{c}\text { Reste venant } \\
\text { durant une venue }\end{array}$ & \\
\hline En attente & Aucune interaction essentielle & $\begin{array}{c}\text { Se tient hors de l'espace } \\
\text { de combat }\end{array}$ & $\begin{array}{l}\text { Attend son tour } \\
\text { pour affronter le } \\
\text { défenseur }\end{array}$ & \\
\hline
\end{tabular}

Tableau 3 : Rôles sociomoteurs au jeu de prix de Lille.

Pour les jeux d'armes, j'ai repris comme mentionné plus haut les rôles qui se détachent du règlement de Lille.

Il est possible au cours du jeu de passer d'un rôle à l'autre. Le diagramme de changement de rôle est un graphe orienté permettant de représenter visuellement les possibilités qu'offre chaque jeu de modifier les contraintes auxquelles le joueur est astreint.

Chaque rôle est représenté par un cercle; les flèches signalent les possibilités de changement de rôle entre deux phases élémentaires du jeu.

$\mathrm{Au}$ jeu des barres, une phase élémentaire est marquée soit par la capture d'un joueur, soit par la libération des prisonniers.

$\mathrm{Au}$ jeu de paume, la phase élémentaire commence au service et se termine au gain d'un point, appelé un "quinze »; il est constitué d'un ou deux services s'il y a chasse. Chaque phase élémentaire s'inscrit dans un cycle plus long, le "jeu », qui se termine dès qu'un joueur a gagné quatre points. Le décompte de marques s'effectue donc de manière similaire au tennis, c'est-à-dire «quinze », « trente », « quarante-cinq », « jeu ».

$\mathrm{Au}$ jeu de prix, la phase élémentaire consiste en une venue d'un défenseur.

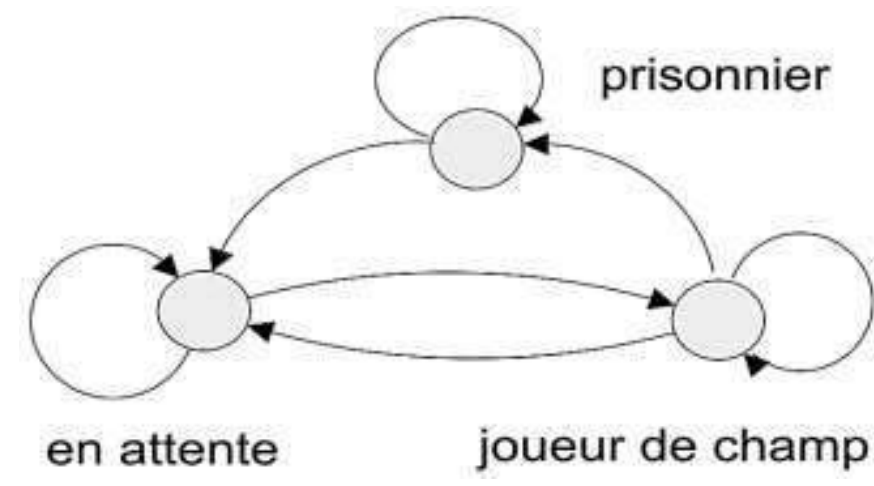

Figure 2: Diagramme de changement de rôle pour le jeu des barres $^{24}$. 


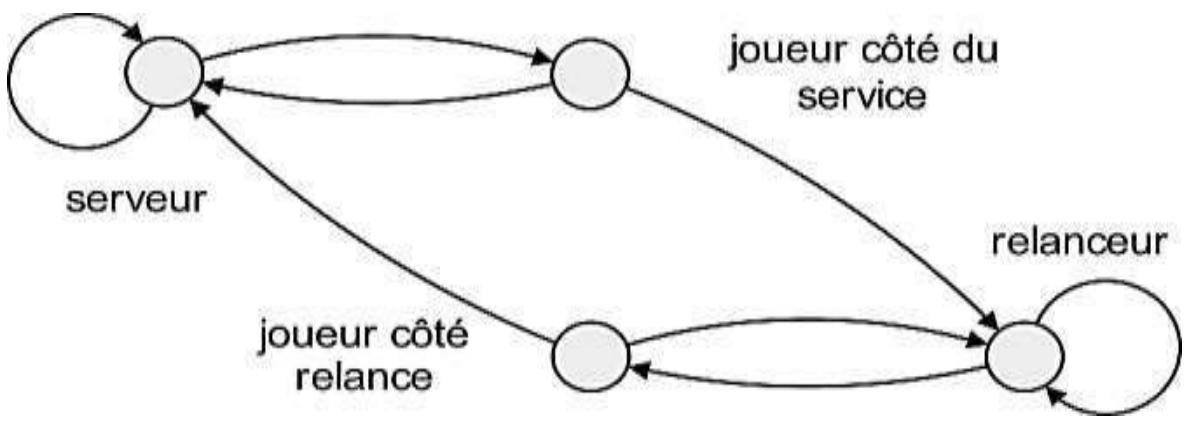

Figure 3 : Diagramme de changement de rôle pour le jeu de courte paume.

$\mathrm{Au}$ sein de chaque rôle sociomoteur, il est possible de retrouver des unités de l'action stratégique qui lui sont propres et qui définissent les sous-rôles sociomoteurs. Ce travail prend son importance dès qu'il est question d'ouvrir l'expérimentation à l'improvisation : quelles sont les tactiques possibles et si possible historiquement attestées ? Il est difficile de dresser une liste exhaustive de ces sous-rôles, d'autant plus que les facteurs culturels influençant ou inhibant la réalisation de certaines tactiques ne sont pas négligeables. Pour le jeu de paume ou le jeu des barres, les contraintes induites par les statuts sociomoteurs ainsi que les récits de parties qui nous sont parvenus permettent de proposer une liste sinon complète, du moins assez précise. Pour l'escrime, l'exercice est plus ardu; les règles sont très ouvertes et les livres d'armes apparaissent comme la source la plus pertinente pour retrouver les sous-rôles sociomoteurs. J'ai repris ici un document récemment redécouvert, le Cabinet d'escrime de l'espée et poingnardt du capitaine Péloquin daté aux alentours de $1600^{25}$, auquel j'ai commencé à appliquer cette analyse. Bien que le travail reste inabouti, les résultats me semblent suffisamment éloquents pour être présentés à ce stade.

\section{Sous-rôles du jeu de paume}

Comme pour les rôles, le détail des sous-rôles est repris pour l'essentiel du travail de Méry.

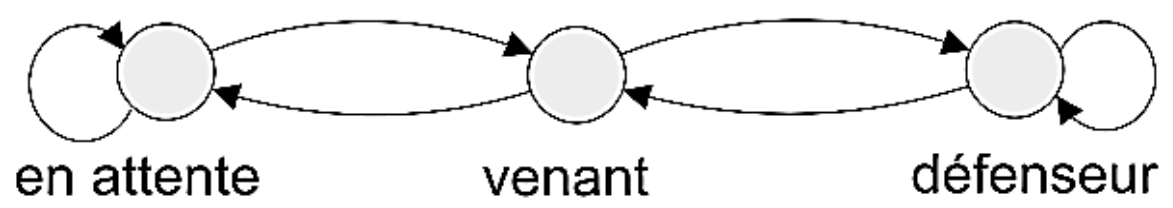

Figure 4 : Diagramme de changement de rôle pour le jeu de prix de Lille. 


\begin{tabular}{|c|c|c|c|c|c|}
\hline \multicolumn{6}{|c|}{ Serveur } \\
\hline $\begin{array}{c}\text { Intention } \\
\text { stratégique }\end{array}$ & Sous-rôles & Autrui & Espace & Temps & Objets \\
\hline $\begin{array}{l}\text { Mettre en } \\
\text { difficulté }\end{array}$ & Sert à plat & $\begin{array}{l}\text { Possibilité de } \\
\text { servir en } \\
\text { fonction du } \\
\text { placement et } \\
\text { de l'attitude }\end{array}$ & $\begin{array}{l}\text { La balle rebondit ou roule sur } \\
\text { le toit de la galerie et sur le } \\
\text { toit du devers (opt.) puis } \\
\text { retombe dans le carré de } \\
\text { service } \\
\text { Les joueurs se tiennent où ils } \\
\text { veulent sur le terrain }\end{array}$ & $\begin{array}{l}\text { Le joueur a du temps } \\
\text { pour se replacer car la } \\
\text { balle atterrit dans le } \\
\text { carré une seconde et } \\
\text { demi après la frappe }\end{array}$ & $\begin{array}{c}\text { Pas d'effet dans } \\
\text { la balle }\end{array}$ \\
\hline Idem & $\begin{array}{c}\text { Slice sur la } \\
\text { droite }\end{array}$ & Idem & Idem & Idem & $\begin{array}{l}\text { Balle travaillée } \\
\text { de droite à } \\
\text { gauche }\end{array}$ \\
\hline Idem & $\begin{array}{c}\text { Slice sur la } \\
\text { gauche }\end{array}$ & Idem & Idem & Idem & $\begin{array}{l}\text { Balle travaillée } \\
\text { de gauche à } \\
\text { droite }\end{array}$ \\
\hline Idem & $\begin{array}{l}\text { Coupé à la } \\
\text { cuillère }\end{array}$ & Idem & Idem & Idem & $\begin{array}{c}\text { Balle travaillée } \\
\text { de gauche à } \\
\text { droite et de bas } \\
\text { en haut }\end{array}$ \\
\hline
\end{tabular}

Tableau 4 : Sous-rôles sociomoteurs du serveur à la courte. 


\begin{tabular}{|c|c|c|c|c|c|}
\hline \multicolumn{6}{|c|}{ Rôle : relanceur } \\
\hline $\begin{array}{l}\text { Faire un } \\
\text { coup gagnant }\end{array}$ & Retour à plat & $\begin{array}{l}\text { Possibilité de } \\
\text { retourner en } \\
\text { fonction du } \\
\text { placement et de } \\
\text { l'attitude }\end{array}$ & $\begin{array}{l}\text { Tente mettre la balle dans } \\
\text { une des galeries ou loin de } \\
\text { l'adversaire dans un } \\
\text { espace ouvert ou réaliser } \\
\text { une chasse }\end{array}$ & $\begin{array}{l}\text { Relanceur prend la } \\
\text { balle très tôt } \\
\text { La préparation du } \\
\text { coup est rapide } \\
\text { Balle rapide }\end{array}$ & $\begin{array}{c}\text { Pas d'effet dans } \\
\text { la balle }\end{array}$ \\
\hline $\begin{array}{c}\text { Faire un } \\
\text { coup gagnant } \\
\text { ou } \\
\text { neutraliser }\end{array}$ & Retour coupé & Idem & $\begin{array}{l}\text { Mettre la balle loin de } \\
\text { l'adversaire dans un } \\
\text { espace ouvert ou réaliser } \\
\text { une chasse } \\
\text { Faire courir la balle sur } \\
\text { les toits }\end{array}$ & $\begin{array}{l}\text { Préparation du coup } \\
\text { rapide }\end{array}$ & $\begin{array}{l}\text { Balle travaillée } \\
\text { de haut en bas }\end{array}$ \\
\hline $\begin{array}{l}\text { Faire un } \\
\text { coup gagnant } \\
\text { ou } \\
\text { ouvrir le } \\
\text { court }\end{array}$ & Retour amorti & $\begin{array}{c}\text { Idem + créer un } \\
\text { effet de } \\
\text { surprise }\end{array}$ & $\begin{array}{l}\text { Mettre la balle le plus près } \\
\text { possible du filet }\end{array}$ & $\begin{array}{l}\text { Temps de réaction } \\
\text { de l'adversaire } \\
\text { surpris va lui laisser } \\
\text { du temps et lui } \\
\text { donner un avantage }\end{array}$ & $\begin{array}{l}\text { Balle travaillée } \\
\text { de haut en bas } \\
\text { avec un retour } \\
\text { prononcé vers le } \\
\text { haut. Préparation } \\
\text { masquée }\end{array}$ \\
\hline
\end{tabular}

Tableau 5 : Sous-rôles sociomoteurs du relanceur à la courte. 


\begin{tabular}{|c|c|c|c|c|c|}
\hline \multicolumn{5}{|c|}{ Rôle : joueur de champ côté service } & Faire un \\
coup gagnant & Frappe à plat & Idem relanceur & $\begin{array}{c}\text { Tente mettre la balle } \\
\text { dans la grille ou loin de } \\
\text { l'adversaire dans un } \\
\text { espace ouvert ou réaliser } \\
\text { une chasse }\end{array}$ & Idem relanceur & Idem relanceur \\
\hline $\begin{array}{c}\text { Faire un } \\
\text { coup gagnant }\end{array}$ & Frappe coupée & Idem relanceur & Idem relanceur & Idem relanceur & Idem relanceur \\
\hline $\begin{array}{c}\text { Faire un } \\
\text { coup gagnant }\end{array}$ & Amorti & Idem relanceur & Idem relanceur & Idem relanceur & Idem relanceur \\
\hline $\begin{array}{c}\text { Faire un } \\
\text { coup gagnant }\end{array}$ & Volée & $\begin{array}{c}\text { Possibilité } \\
\text { retourner en } \\
\text { fonetion } \\
\text { placement et de } \\
\text { l'attitude }\end{array}$ & Mettre balle loin de \\
l'adversaire & $\begin{array}{c}\text { Montée rapide, } \\
\text { balle rapide qui } \\
\text { s'écrase }\end{array}$ & $\begin{array}{c}\text { Frapper la balle } \\
\text { devant de haut } \\
\text { en bas. }\end{array}$ \\
\hline
\end{tabular}

Tableau 6 : Sous-rôles sociomoteurs du joueur de champ côté service à la courte paume. 


\begin{tabular}{|c|c|c|c|c|c|}
\hline \multicolumn{7}{|c|}{ Rôle : joueur de champ côté relance } \\
\hline $\begin{array}{c}\text { Faire un coup } \\
\text { gagnant }\end{array}$ & Frappe à plat & Idem relanceur & Idem relanceur & Idem relanceur & Idem relanceur \\
\hline $\begin{array}{c}\text { Faire un coup } \\
\text { gagnant }\end{array}$ & $\begin{array}{c}\text { Frappe } \\
\text { coupée }\end{array}$ & Idem relanceur & Idem relanceur & Idem relanceur & Idem relanceur \\
\hline $\begin{array}{c}\text { Faire un coup } \\
\text { gagnant }\end{array}$ & Amorti & Idem relanceur & Idem relanceur & Idem relanceur & Idem relanceur \\
\hline $\begin{array}{c}\text { Faire un coup } \\
\text { gagnant }\end{array}$ & Volée & $\begin{array}{c}\text { Possibilité } \\
\text { retourner en } \\
\text { fonction } \\
\text { placement et de } \\
\text { lattitude }\end{array}$ & $\begin{array}{c}\text { Mettre balle loin de } \\
\text { l'adversaire }\end{array}$ & $\begin{array}{c}\text { Montée rapide, } \\
\text { balle rapide qui } \\
\text { s'écrase }\end{array}$ & $\begin{array}{c}\text { Frapper la balle } \\
\text { devant de haut en } \\
\text { bas. }\end{array}$ \\
\hline
\end{tabular}

Tableau 7 : Sous-rôles sociomoteurs du joueur de champ côté relance à la courte paume. 


\begin{tabular}{|c|c|c|c|c|}
\hline Rỏle / sous-rôle & Intention stratégique & Autrui & Espace & Temps \\
\hline Prisonnier & & Peut être délivré & $\begin{array}{l}\text { À côté du camp } \\
\text { adverse }\end{array}$ & $\begin{array}{c}\text { Délivré après qu'un } \\
\text { partenaire ait touché le } \\
\text { dernier dans la chaîne des } \\
\text { prisonniers }\end{array}$ \\
\hline $\begin{array}{l}\text { En attente / } \\
\text { observateur }\end{array}$ & & $\begin{array}{l}\text { Ne peut être capturé, ni capturer ni } \\
\text { libérer. }\end{array}$ & Dans son camp & Attend son tour \\
\hline $\begin{array}{l}\text { En attente / } \\
\text { défié }\end{array}$ & & Tend la main & Dans son camp & $\begin{array}{l}\text { Attend d'être défié par un } \\
\text { adversaire par une tape sur } \\
\text { la main }\end{array}$ \\
\hline $\begin{array}{l}\text { En attente / } \\
\text { démarreur }\end{array}$ & Entrer sur le terrain & $\begin{array}{c}\text { Annonce "j'ai barre sur } \mathrm{X}^{\prime} \\
\text { A barre sur X }\end{array}$ & Dans son camp & $\begin{array}{l}\text { Ne peut entrer qu'après } \\
\text { lentrée de } \mathrm{X} \text { dans le terrain }\end{array}$ \\
\hline $\begin{array}{l}\text { Joueur de champ } \\
\text { / défieur }\end{array}$ & Initier l'unité de jeu & $\begin{array}{l}\text { Défie un adversaire en lui frappant } \\
\text { dans la main }\end{array}$ & $\begin{array}{l}\text { Seul dans le } \\
\text { terrain de jeu }\end{array}$ & $\begin{array}{l}\text { Première action d'une unité } \\
\text { de jeu }\end{array}$ \\
\hline $\begin{array}{l}\text { Joueur de champ } \\
\text { / poursuivi }\end{array}$ & $\begin{array}{l}\text { Ne pas être capturé. } \\
\text { Provoquer la capture de } \\
\text { l'adversaire en lapprochant de } \\
\text { celui qui a barre sur lui. }\end{array}$ & $\begin{array}{c}\text { Est poursuivit et tente de } \\
\text { s'échapper de l'unique adversaire } \\
\text { qui a barre sur lui. } \\
\text { Peut avoir barre sur un adversaire. }\end{array}$ & $\begin{array}{c}\text { Dans le terrain de } \\
\text { jeu }\end{array}$ & \\
\hline $\begin{array}{l}\text { Joueur de champ } \\
\text { / poursuivant }\end{array}$ & $\begin{array}{c}\text { Se rapprocher de sa cible pour } \\
\text { permettre sa capture. } \\
\text { Forcer la cible à s'éloigner de la } \\
\text { personne. } \\
\text { Faire un prisonnier }\end{array}$ & $\begin{array}{c}\text { Poursuit l'unique adversaire sur } \\
\text { lequel il a barre et tente de le } \\
\text { toncher dès qu'il est à distance. } \\
\text { Peut être sous les barres d'un autre } \\
\text { adversaire. }\end{array}$ & $\begin{array}{c}\text { Dans le terrain de } \\
\text { jeu }\end{array}$ & \\
\hline $\begin{array}{l}\text { Joueur de champ } \\
\text { / délivreur }\end{array}$ & $\begin{array}{l}\text { Libérer les prisonniers. } \\
\text { Si poursuivi, permet d'éviter la } \\
\text { capture }\end{array}$ & $\begin{array}{c}\text { Tente de toucher la main du dernier } \\
\text { prisonnier de la chaîne pour les } \\
\text { libérer. } \\
\text { Peut étre sous les barres d'un autre } \\
\text { adversaire. }\end{array}$ & $\begin{array}{c}\text { Dans le terrain de } \\
\text { jeu }\end{array}$ & \\
\hline
\end{tabular}

Tableau 8 : Sous-rôles sociomoteurs au jeu des barres. 


\section{Sous-rôles du jeu des barres}

Les contraintes existant de par les règles entre les différentes phases de jeu permettent de représenter un diagramme des changements de sousrôles pour le jeu des barres.

Chaque sous-rôle est représenté par un cercle plein ; les rôles sont rappelés par un cercle en pointillé entourant l'ensemble de ses sous-rôles ; les flèches signalent les possibilités de changement de sous-rôle entre deux phases élémentaires du jeu.

\section{Sous-rôles du jeu d'épée et poignard}

La liste de sous-rôle présentée ci-dessous n'est

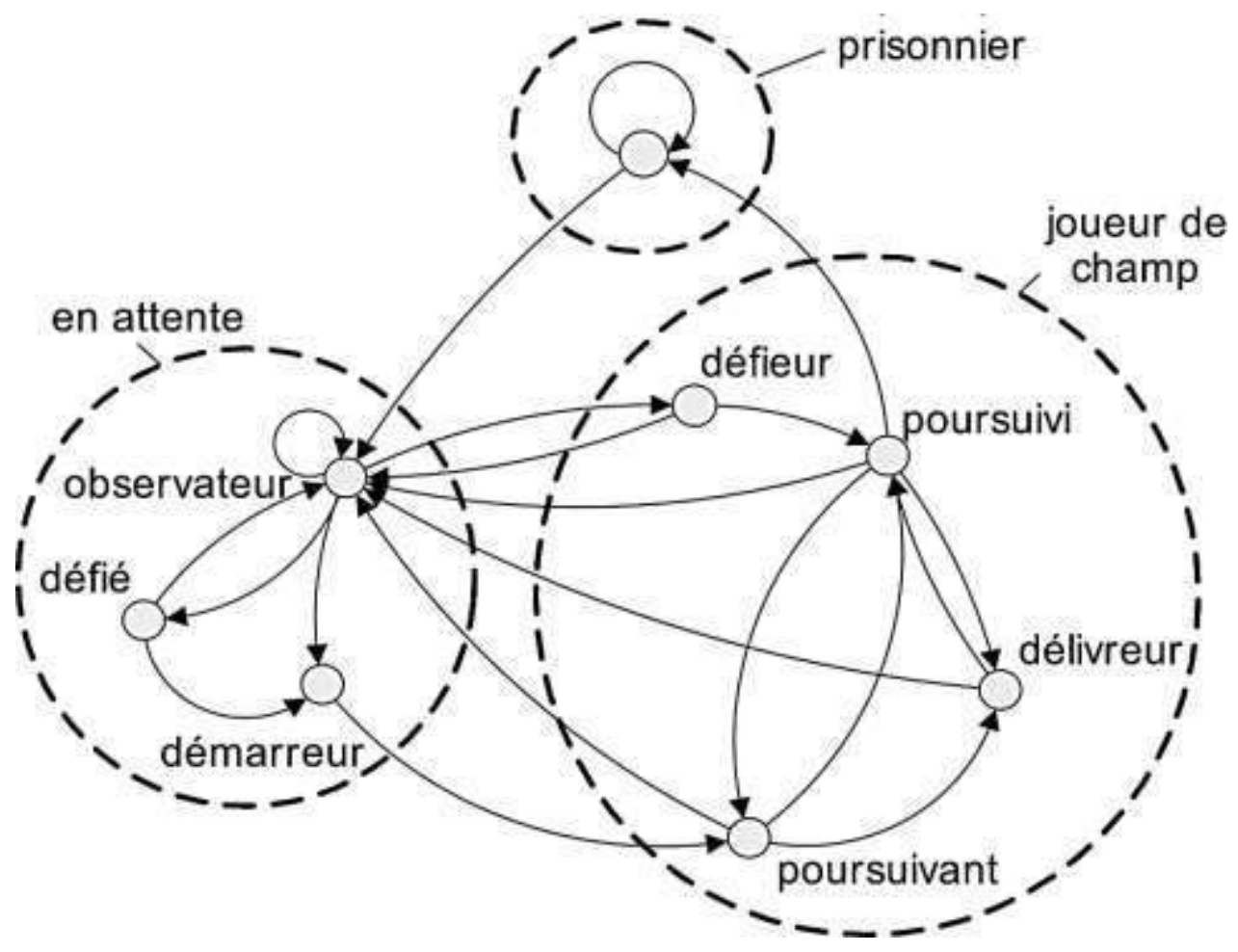

Figure 5 : Diagramme de changement de sous-rôle au jeu des barres. 


\begin{tabular}{|c|c|c|c|c|c|}
\hline Sous-rôles & $\begin{array}{l}\text { Intention } \\
\text { stratégique }\end{array}$ & Autrui & Temps & Ses objets & Objets d'autrui \\
\hline $\begin{array}{l}\text { Frapper de pied } \\
\text { ferme }\end{array}$ & Toucher & Proche & $\begin{array}{l}\text { Dès le début } \\
\text { (primer), ou } \\
\text { après une feinte } \\
\text { ou une batterie }\end{array}$ & $\begin{array}{l}\text { La pointe va à la } \\
\text { cible la plus proche }\end{array}$ & $\begin{array}{l}\text { Le poignard sert à } \\
\text { écarter l'arme adverse } \\
\text { si nécessaire }\end{array}$ \\
\hline $\begin{array}{c}\text { Première (attaque } \\
\text { d'estoc avec un } \\
\text { pas) }\end{array}$ & Toucher & $\begin{array}{c}\text { À distance } \\
\text { d'engagement }\end{array}$ & $\begin{array}{l}\text { Préparation du } \\
\text { coup rapide }\end{array}$ & $\begin{array}{l}\text { La pointe va à la } \\
\text { cible la plus proche }\end{array}$ & $\begin{array}{l}\text { Le poignard sert à } \\
\text { écarter l'arme adverse } \\
\text { si nécessaire }\end{array}$ \\
\hline $\begin{array}{l}\text { Frapper d'un } \\
\text { estramaçon }\end{array}$ & Toucher & Proche & $\begin{array}{l}\text { Après une } \\
\text { batterie }\end{array}$ & $\begin{array}{c}\text { L'épée en } \\
\text { déplacement sur le } \\
\text { côté }\end{array}$ & $\begin{array}{l}\text { La pointe de l'épée } \\
\text { vers le côté, l'épée } \\
\text { éventuellement gardée } \\
\text { par le poignard }\end{array}$ \\
\hline Tirade & $\begin{array}{c}\text { Mettre en } \\
\text { difficulté (feinte) }\end{array}$ & $\begin{array}{c}\text { À distance } \\
\text { d'engagement }\end{array}$ & & $\begin{array}{l}\text { La pointe va vers la } \\
\text { cible la plus proche } \\
\text { avant d'être retirée }\end{array}$ & \\
\hline Cavement & $\begin{array}{l}\text { Mettre en } \\
\text { difficulté }\end{array}$ & $\begin{array}{c}\text { À distance } \\
\text { d'engagement }\end{array}$ & & $\begin{array}{c}\text { La pointe va vers la } \\
\text { cible la plus proche } \\
\text { avant de tourner sous } \\
\text { les armes pour } \\
\text { présenter la pointe de } \\
\text { bas vers le haut } \\
\end{array}$ & \\
\hline Voûtement & $\begin{array}{l}\text { Mettre en } \\
\text { difficulté }\end{array}$ & $\begin{array}{c}\text { À distance } \\
\text { d'engagement }\end{array}$ & & $\begin{array}{c}\text { La pointe va vers la } \\
\text { cible la plus proche } \\
\text { avant de tourner sous } \\
\text { les armes pour } \\
\text { présenter la pointe de } \\
\text { haut vers le bas } \\
\end{array}$ & \\
\hline Batterie & $\begin{array}{c}\text { Pousser la pointe } \\
\text { adverse de côté } \\
\text { ou se défendre } \\
\text { d'un estoc }\end{array}$ & $\begin{array}{c}\text { À distance } \\
\text { d'engagement }\end{array}$ & $\begin{array}{l}\text { Préparation du } \\
\text { coup rapide }\end{array}$ & $\begin{array}{l}\text { L'épée va frapper du } \\
\text { faible le faible de } \\
\text { l'épée adverse }\end{array}$ & $\begin{array}{l}\text { La pointe de l'épée } \\
\text { dirigée vers soi, } \\
\text { éventuellement } \\
\text { s'approchant vivement }\end{array}$ \\
\hline$\ldots$ & $\ldots$ & $\ldots$ & $\ldots$ & $\ldots$ & $\ldots$ \\
\hline
\end{tabular}

Tableau 9 : Sous-rôles sociomoteurs dans le traité d'escrime à l'épée et poignard de Péloquin.

pas exhaustive; elle s'est construite non pas sur l'observation de pratiquants, mais déduits d'un livre d'armes attribué au capitaine Péloquin. La décomposition en sous-rôle présente l'intérêt de proposer une vision synthétique pouvant servir à la comparaison entre le contenu de différents livres d'armes, voire de servir à bâtir une synthèse plus complète pour un ensemble de livres d'armes cohérent. 
Lors du changement de sous-rôle sociomoteur, le joueur modifie nécessairement sa conduite motrice informant ainsi, consciemment ou non, ses partenaires et adversaires. L'association du comportement observable et du projet tactique perçu constitue un «praxème » 26 . Celui-ci fait nécessairement partie intégrante d'une action en train de se dérouler et compose un indice permettant l'anticipation motrice. Chaque personne peut attribuer une valeur de praxème différente à une séquence comportementale donnée selon son expérience et sa capacité à décoder les signes décelés dans le comportement d'autrui ; dans le cas du jeu d'opposition, la feinte consiste à émettre des contre-signaux de sorte à perturber le travail d'analyse de son adversaire. L'étude des praxèmes dans les traités techniques permet de dresser une liste des projets tactiques ou sous-rôles sociomoteurs, ce qui n'est pas toujours aisé à repérer. En effet, le texte se contente souvent de ne donner que le comportement observable ( « l'attaquant vous attaque avec un coup de haut en bas ») et il est rare de trouver une mention explicite d'un praxème, comme dans l'exemple suivant extrait du livre d'armes de Péloquin : "Quand vous voiez que vostre ennemy a son espée en dedans de vostre poingnardt le vous baillant expressement en prise pour vous faire le coup de poingnardt... ${ }^{27}$.

\section{Conclusion}

À travers l'étude sociomotrice de ces trois exemples de jeux sportifs, il est possible de proposer des éléments de réponse à la différence de traitement scriptural des codes verbaux entre ceux-ci. Cette forte dissymétrie peut s'entendre par le raisonnement suivant: tout d'abord les barres n'imposent ni structure ni matériel nécessitant l'établissement de codes, de règles, de valeurs de références, ce qui le différencie de certaines formes du jeu de paume, en particulier de la courte paume. Le fait que pour cette dernière de fortes sommes d'argent pouvaient être en jeu, parallèlement à la complexité des caractéristiques des lieux, peut expliquer la mise par écrit des normes. Par contre les jeux d'armes se distinguent des précédents par le fait que les aptitudes motrices acquises au cours du jeu sont supposées et réputées se transposer pour défendre sa vie. Cette proximité avec un contexte peu normé et réglementé est probablement en cause dans la production d'ouvrages de perfectionnement.

L'étude des rôles et sous-rôles sociomoteurs permet de mettre en exergue les interactions motrices et impose de contextualiser le geste, non seulement vis-à-vis de l'environnement direct, mais aussi, et surtout vis-à-vis d'autrui, homme... ou cheval. Cette étude offre enfin un canevas permettant la comparaison entre différentes activités motrices ainsi qu'entre différentes sources pour un même type d'activités (par exemple entre deux traités d'escrime). Les notions de praxèmes, rôles et sous rôles sociomoteurs ne se limitent pas aux seuls jeux sportifs pour lesquels elles ont été élaborées et précisées par Parlebas; elles doivent pouvoir être étendues à un plus grand ensemble de situations de sociomotricités.

1 PARLEBAS Pierre, Jeux, sports et sociétés. Lexique de praxéologie motrice, Paris, INSEP, 1998, p. 324-335 et p. 341343 .

2 Pour simplifier, la sémiotricité est l'application de la sémiologie aux situations motrices. Pour une définition plus complète, voir PARLEBAS Pierre, op. cit., 1998, p. 324.

3 MEHL Jean-Michel, Les jeux au royaume de France du XIII au XVI ${ }^{e}$ siècle, Paris, Fayard, 1990, p. 183-226.

4 VAUCELLE Serge, L'art de jouer à la cour, transformation des jeux d'exercice dans l'éducation de la noblesse française au début de l'ère moderne (XIII ${ }^{e}-X V I I^{e}$ siècles), thèse de doctorat de l'EHESS, 2004. p. 39.

5 MEHL Jean-Michel, Les jeux au royaume de France du XIII au XVI ${ }^{e}$ siècle, Paris, Fayard, 1990, p. 63-64. 
6 SERNA Pierre, "L'art de tuer comme art de vivre ", Croiser le fer, violence et culture de l'épée dans la France moderne (XVIeXVIII ${ }^{e}$ siècle), Pascal Brioist, Hervé Drévillon, Pierre Serna éd., Seyssel, Champ Vallon, 2001, p. 199-237.

7 SCRIVE-BERTIN, «La confrérie d'armes de S. Michel ou des escrimeurs lillois », Bulletin de la Commission historique du département du Nord, t. 19, 1890, p. 106-110.

8 LACHET Claude, « De la guerre à la table ronde : variations sémantiques des locutions cil dedens et cil dehors ", Le tournoi au Moyen Age, Gonthier Nicole (éd.), Cahiers du Centre d'Histoire Médiévale $\mathrm{N}^{\circ}$, Lyon : Université Jean Moulin Lyon 3, p. 59-73.

9 MEHL Jean-Michel, op. cit., 1990, p. 31-48.

10 MERY Sthéphane, Un filet et des sports, Approches sociologique, historique, prospective, comportementaliste, Paris, Harmattan, 2007. p. 280-290.

11 Ce développement est particulièrement aisé à suivre par l'implantation des salles de paume dans la plupart des grandes villes. Par exemple, pour le Saint-Empire, voir GILLMEISTER Heiner, « Der Topspin taugte nichts im alten Jeu de la Paume: das Tennisspiel in drei Jahrhunderten (1500-1800) », Bewegtes Leben. Körpertechniken in der Frühen Neuzeit, Rebekka. von Mallinckrodt éd., Wolfenbüttel, 2008, p. 205-229. Pour l'Espagne voir : NANTEUIL Edmond de, SAINT-CLAIR G. de, DELAHAYE C., La Paume et le Lawn-tennis, Paris, Hachette, 1898. p. 390. Il n'est pas aisé de savoir si les formes populaire du jeu de paume eurent le même succès, d'une part du fait de la modestie des pratiquants, mais aussi l'absence de lieu spécifique dédié à ce loisir.

12 MEHL Jean-Michel, op. cit., 1990, p. 65-68.

13 Mémoires d'Olivier de la Marche, Paris, 1785, p. 279.

14 MEHL Jean-Michel, op. cit., 1990, p. 65-68.

15 Histoire de la révolution de Gênes, Paris, Chaignieau aîné, 1797, p. 19-22.

16 Encyclopédie méthodique ou par ordre de matière, Art militaire, t. 4, Paris, 1797, p. 67.

17 BASCETTA Carlo, « Les codes verbaux de jeu et les sources de la littérature sportive italienne du XVe au XVII ${ }^{\mathrm{e}}$ siècle » Les jeux à la Renaissance, Jean-Claude Philippe Ariès, Jean-Claude Margolin (éd)., Vrin, 1982, p. 95-104.

18 Règles du jeu des Barres, dressé et imprimé pour le Roy, Paris : Collombat, 1719.4 pages. Mentionné dans : L'imprimerie du cabinet du roi au château des Tuileries sous Louis XV (17181730) dans Bulletin de la Société de l'histoire de Paris et de l'Ile-
de-France, Tome 18. Paris : Honoré Champion, 1891, p. 35-45. Ce document n'a pu être retrouvé ni consulté.

19 SCAINO DA SALO Antonio, Trattato del giuoco della palla, Urbino, QuatroVenti, 2000.

20 SAINT-DIDIER Henri de, "Traité sur l'exercice, et certains points requis de scavoir au jeu de paulme... », Traicté contenant les secrets du premier livre sur l'espée seule, Paris, Mathurin Challenge, 1573 , fol. $87 \mathrm{r}^{\circ}-89 \mathrm{v}^{\circ}$.

21 PARLEBAS Pierre, "Mathématisation élémentaire de l'action dans les jeux sportifs ", Mathématiques et sciences humaines, t. 170, 2005, p. 95-117.

22 PARLEBAS Pierre, "Modélisation dans les jeux et les sports », Mathématiques et sciences humaines, t. 170, 2005, p. 11-45.

23 Légèrement adapté à partir de celui présenté dans MERY Stéphane, 2007, op. cit, p. 118-119. J'ai séparé le rôle de joueur d'échange en distinguant le côté puisque cela influe directement sur le placement dans le cours.

24 Le diagramme de changement de rôle du jeu des barres dans sa version moderne est décrite dans PARLEBAS Pierre, "Conduites motrices et communication dans les jeux », Langage : langue parlée - langue écrite et créativité à l'école maternelle, brochure $\mathrm{n}^{\circ}$ 2365, Paris : INRDP, 1974, p. 221-235.

25 Manuscrit KW 73J39 de la bibliothèque royale des Pays-Bas.

26 Il s'agit de toute conduite motrice d'un joueur, susceptible d'être interprétée comme un signe par les autres participants. Le "signifiant » en est le comportement observable, et le "signifié » le projet tactique correspondant tel qu'il est perçu par les autres. Pour une définition plus complète, voir PARLEBAS Pierre, op. cit., 1998, p. 260.

27 Manuscrit KW 73J39 de la bibliothèque royale des Pays-Bas, fol. $26 \mathrm{v}^{\circ}$. 